\title{
Ultrafast dynamics of magnetic domain structures probed by coherent Free-Electron Laser light
}
L. Müller, ${ }^{1}$ S. Schleitzer, ${ }^{1}$ C. Gutt,,${ }^{1,2}$
B. Pfau, ${ }^{3}$ S. Schaffert, ${ }^{3}$ J. Geilhufe, ${ }^{4}$ C. von Korff Schmising, ${ }^{3}$ M. Schneider, ${ }^{3}$ C. M. Günther, ${ }^{3}$ F. Büttner, ${ }^{3,5}$ F. Capotondi, ${ }^{6}$ E. Pedersoli, ${ }^{6}$ S. Düsterer, ${ }^{1}$ H. Redlin, ${ }^{1}$ A. Al-Shemmary, ${ }^{1}$ R. Treusch, ${ }^{1}$ J. Bach, ${ }^{7}$ R. Frömter, ${ }^{7,2}$ B. Vodungbo, ${ }^{8}$ J. Gautier,${ }^{8}$ P. Zeitoun, ${ }^{8}$ H. Popescu, ${ }^{9}$ V. Lopez-Flores, ${ }^{9}$ N. Beaulieu, ${ }^{9}$ F. Sirotti, ${ }^{9}$ N. Jaouen, ${ }^{9}$ G. Malinowski, ${ }^{10}$ B. Tudu,,${ }^{11}$ K. Li, ${ }^{11}$ J. Lüning, ${ }^{11}$ H. P. Oepen, ${ }^{7,2}$ M. Kiskinova, ${ }^{6}$ S. Eisebitt, ${ }^{3,4}$ and G. Grübel ${ }^{1,2}$

${ }^{1}$ Deutsches Elektronen-Synchrotron DESY, 22607 Hamburg, Germany

${ }^{2}$ The Hamburg Centre for Ultrafast Imaging, 22761 Hamburg, Germany

${ }^{3}$ Institut für Optik und Atomare Physik, TU Berlin, 10623 Berlin, Germany

${ }^{4}$ Helmholtz-Zentrum Berlin für Materialien und Energie GmbH, 14109 Berlin, Germany

${ }^{5}$ Institute of Physics, Johannes Gutenberg-Universität Mainz, 55099 Mainz, Germany

${ }^{6}$ FERMI, Elettra-Sincrotrone Trieste, 34149 Basovizza, Trieste, Italy

${ }^{7}$ Institut fr angewandte Physik, Universität Hamburg, 20355 Hamburg, Germany

${ }^{8}$ Laboratoire d'Optique Appliqué, ENSTA ParisTech-CNRS UMR 7639-École polytechnique, 91761 Palaiseau, France.

${ }^{9}$ Synchrotron SOLEIL, F-91192 Gif Sur Yvette, France

${ }^{10}$ Laboratoire de Physique des Solides, CNRS-UMR 8502, Université Paris-Sud, F-91405 Orsay, France

${ }^{11}$ Laboratoire de Chimie Physique Matière et Rayonnement-CNRS UMR 7614, Université Pierre et Marie Curie, 75005 Paris, France 


\section{INTRODUCTION}

The free-electron laser (FEL) sources FLASH in Hamburg, LCLS at Stanford and FERMI in Trieste provide XUV to soft x-ray radiation (FLASH and FERMI) or soft to hard $\mathrm{x}$-ray radiation (LCLS) with unprecedented parameters in terms of ultrashort pulse length, high photon flux, and coherence. These properties make FELs ideal tools for studying ultrafast dynamics in matter on a previously unaccessible level. This paper reviews first results obtained at FEL sources during the last years in the field of magnetism research. We start with pioneering experiments at FLASH demonstrating the feasibility of magnetic scattering at FELs [1, 2], then present pump-probe scattering experiments [3, 4] as well as the first FEL magnetic imaging experiments [5], and finally discuss a limitation of the scattering methods due to a quenching of the magnetic scattering signal by high-fluence FEL pulses [6]. All of the presented experiments exploit the $x$-ray magnetic circular dichroism effect $[7,8]$ to obtain element-specific magnetic scattering contrast, as known from synchrotron experiments [9-12].

One of the key problems in modern magnetism research, ultrafast demagnetization discovered by Beaurepaire in 1996 [13], acts on time scales of a few $100 \mathrm{fs}$, which are not accessible at standard synchrotron radiation sources. Since its discovery, ultrafast demagnetization was studied using optical femtosecond lasers, for a review see e. $\mathrm{g}$. [14], and femtoslicing sources [15] at storage ring sources [16, 17]. In both cases, spatial resolution is limited due to wavelength or flux limitations. In contrast, FELs combine high time resolution, element selectivity, and the spatial resolving power of $x-$ rays with high photon flux and excellent coherence properties. FEL sources, therefore, promise a pivotal step forward in the investigation of ultrafast phenomena on nanometer length scales. The rapid development of the FELs with respect to even shorter pulses,

increased pulse stability, and special double-pulse schemes, e. g. for ultrafast movies [18], will open up exciting new opportunities in the field of material science.

\section{RESONANT SCATTERING FROM MAGNETIC DOMAIN SYSTEMS}

The very first proof-of-principle resonant magnetic scattering experiment at $\mathrm{x}$-ray FEL sources was carried out at FLASH in Hamburg [1]. In 2009 FLASH covered a photon energy range in the fundamental from 6.5 up to $50 \mathrm{~nm}$ with the routinely used cobalt $L_{3^{-}}$ edge at $1.59 \mathrm{~nm}$ out of reach. However, by using the higher harmonics of the radiation 
shorter wave length can be reached on the expense of photon flux. In this experiment FLASH was fine tuned to a fundamental wave length of $7.97 \mathrm{~nm}$ which results in the fifth harmonic located at the cobalt $L_{3}$-edge. The scattering pattern from magnetic domains of a cobalt/palladium multilayer system with a perpendicular magnetic anisotropy and typical domain widths in the $100 \mathrm{~nm}$ range was recorded. This demonstrated that magnetic scattering as it is performed at synchrotron radiation sources is feasible at FELs. However, the fifth harmonic of the undulator makes up only about $0.03 \%$ of the total radiation power and also the beamline optics were not optimized for that photon energy. As a result, only $7 \times 10^{3}$ photons per pulse were available and the scattering signal from multiple FEL pulses had to be accumulated.

Due to this limitation, it was tested if such magnetic domain systems could also be probed at the $M$-edge of cobalt. The required photon energy can be delivered by FLASH using the fundamental wave length of the undulator. Tuning the photon energy to 59.6 $\mathrm{eV}$ corresponding to the Co $M_{2,3}$ resonance yielded magnetic scattering contrast and the experiments at FLASH showed that magnetic domain systems can be non-destructively probed by single FEL pulses [2].

Pulse intensities of 1-2 $\mu \mathrm{J}$ and a large beam size of $250 \mu \mathrm{m}$, yielding a fluence in the range of a few $\mathrm{mJ} / \mathrm{cm}^{2}$, have proven to result in scattering patterns with good statistics (figure 1).

For low-fluence pulses around 1-2 $\mathrm{mJ} / \mathrm{cm}^{2}$, no changes are induced by the FEL [2], enabling measurements of equilibrium dynamics, e. g. in a future double-FEL-pulse experiment. For fluences of $4-5 \mathrm{~mJ} / \mathrm{cm}^{2}$, permanent changes are induced in the domain configuration, such that the average domain width decreases. Here, it is important to note that these changes happen on longer time scales than the FEL pulse duration. The decrease in domain width is caused by a change of the magnetic anisotropy of the multilayer system due to changes in its structure. Scanning-electron micrographs (figure 2) show that the FEL irradiation led to a recrystallization of the system.

We note that owing to the large beam size, data can be taken non-destructively, however, the speckle pattern has a very fine structure with a typical size of $4 \mu \mathrm{m}$ that cannot be resolved by the detector and the information about the local domain structure is lost.

In order to investigate ultrafast demagnetization using FELs, a dedicated sample chamber was constructed allowing for optical-pump-FEL-probe measurements (figure 3) at FLASH [19]. By employing the optical femtosecond laser available at FLASH, it was possible to probe the magnetic-domain system during the de- and remagnetization process [3]. 
Surprisingly, a shift in the peak intensity position of the small-angle scattering ring caused by the maze domain structure towards smaller momentum transfer $q$ was observed on the same time scale of 300 fs as the demagnetization itself (figure 4). Explanations involving a shift of domain walls fail since such processes would imply domain-wall speeds in excess of $10^{7} \mathrm{~m} / \mathrm{s}$. This speed exceeds the group and phase velocity of magnons [20], or domain-wall speeds due to spin currents or magnetic fields $[21,22]$.

The authors of Ref. [3] identify ultrafast superdiffusive spin transport as proposed by Battiato and co-workers [23] as the origin for the shift. Majority and minority-spin electrons have different scattering probabilities in the magnetic system, such that when majority electrons cross a domain wall and thus become minority electrons, their probability to be scattered increases. Hence, minority electrons accumulate in the domain wall region which leads to an effective softening of the domain walls. This softening of the domain walls affects the scattering factor of the domain structure in a way that the small-angle scattering ring shifts to smaller scattering angles. This behavior could be reproduced by Monte-Carlo simulations.

Such experiments show that lateral ultrafast spin transport phenomena play a role in ultrafast demagnetization processes and have to be considered in nano-structured magnetic materials.

When LCLS at SLAC came online, experiments at higher photon energies became possible with high flux allowing for single-shot data acquisition. Using elliptically polarized x-rays, provided by a transmission polarizer [24], at energies of $707 \mathrm{eV}$ ( $\mathrm{Fe} \mathrm{L}_{3^{-}}$ edge) and $1189 \mathrm{eV}$ (Gd $M_{5}$-edge) a nanoscopically inhomogeneous GdFeCo alloy was investigated in an optical-pump - FEL-probe scattering experiment [4]. In this study it was possible to follow the nanoscale transfer of angular momentum after an optical excitation. Making use of the element selectivity and spatial resolving power of the $x$ rays the complex behavior of this transfer and the importance of the inhomogeneities, i.e. gadolinium or iron rich regions, in this material system was demonstrated.

These results show that FELs techniques can play a key role in studying fundamental phenomena in the field of ultrafast magnetization dynamics and spintronics. 


\section{IMAGING OF MAGNETIC DOMAINS}

Especially in view of the results gained in the pump-probe scattering experiments revealing lateral structural changes in the spin system, it is highly desirable to directly image magnetic-domain dynamics at FEL sources with femtosecond time resolution. Lensless Fourier transform holography (FTH) was chosen as imaging technique to take snapshots of the magnetic-domain system.

For the case of magnetic samples, x-ray FTH was first demonstrated in 2004 using synchrotron radiation [10]. From an experimental point of view, this technique is easy to implement as no additional optical elements are needed. Instead, a nano structured mask fabricated directly on the sample substrate defines a field of view of a few micrometers and a nearby pinhole creates a spherical reference wave. For magnetic systems with domains parallel and antiparallel to the beam axis, reference and magnetically scattered object wave fields interfere only if circularly polarized light is used [25]. To date, circularly polarized FEL radiation directly delivered by the source is only available at FERMI@Elettra, Trieste. All other FELs need an additional polarizer to deliver elliptically polarized light at the cost of pulse intensity. The image contrast is diminished by the achivable limited degree of circular polarization.

FTH at FEL sources has been pioneered at LCLS, Stanford, where at the SXR beamline [26] an additional transmission polarizer [24] was used to generate 58\% circularly polarized light with an energy of $778 \mathrm{eV}$ corresponding to the $L_{3}$ edge of cobalt. In this configuration, single-shot holograms were recorded showing a resolution of 80 $\mathrm{nm}[5]$.

After the FEL source FERMI came into operation in 2012 its ability of providing fully circularly polarized radiation was exploited for further experiments and the first magnetic imaging studies have been realized at the diffraction and projection imaging (DiProl) end station of FERMI $[27,28]$. The photon energy has been tuned to the Co M-edge and the undulator has been set to deliver either left or right circularly polarized radiation. Holograms were taken in non-destructive mode. For both helicities, 15850 shots with a fluence around $6 \mu \mathrm{J} / \mathrm{cm}^{2}$ were summed up to gain holograms with sufficient photon counting statistics. The sample design with the magnetic multilayer and holographic mask was optimized for the low photon energy. 
The difference of the scattering patterns from both helicities yields the magnetization hologram (figure 5 a) showing the typical interference structure from the mask geometry and the magnetic speckles. The real-space structure (figure $5 \mathrm{~b}$ ) is reconstructed from the hologram by a single 2D Fourier transform and applying procedures especially adapted to scattering into relatively large angles at a wave length of $20.8 \mathrm{~nm}$ [29]. Domains with a width of $70 \mathrm{~nm}$ are clearly resolved.

A first single-shot hologram of a magnetic domain system has been obtained [27]. However, it was not possible to reach as high resolution as for the multi-shot hologram shown here.

\section{HIGH-FLUENCE LIMIT}

In order to obtain single-shot scattering patterns with excellent photon statistics that take advantage of the coherence of the FEL beam, i. e. speckles are resolved, the beam size has to be decreased and the FEL fluence has to be increased in contrast to what was used in the scattering experiments discussed previously. From other FEL imaging experiments, e.g. [30] it was expected, that the sample is destroyed due to the resulting high fluence on longer time scales, while the FEL pulse is short enough to probe the still unperturbed (magnetic) state of the sample.

Yet, the experiment shows a different behavior with respect to the scattering signal recorded: While a low-fluence multi-shot reference measurement features the expected scattering pattern (figure 6 a), the corresponding single-shot pattern shows less scattering intensity and also a lack of speckle contrast [6]. During the experiment, only the pulse energy was altered by inserting solid-state absorbers; the photon energy of $59.6 \mathrm{eV}$ and the pulse length of $100 \mathrm{fs}$ stayed unchanged.

It is argued in Ref. [6] that the intensity reduction by a factor of about 20 is a consequence of photon absorption. Photoelectrons are created and as a consequence the binding energy of the remaining $3 p$ electrons increases such that the energy of the incident photons is no longer suitable for the resonant scattering process.

To corroborate this idea, a calculation of the electronic structure using a dedicated Hartree-Fock-Slater model code [31] has been performed. This study finds that, already after 10-15 fs more ionized than non-ionized cobalt atoms are present in the sample, 
leading to a shift in the absorption edge.

This effect implies a photon flux limitation for resonant magnetic scattering at low photon energies.

\section{SUMMARY AND OUTLOOK}

The advent of FEL sources makes it now possible to study ultrafast magnetization dynamics in an element selective and spatially resolved way. A spatial response was seen during ultrafast demagnetization, implying that superdiffusive spin transport plays an important role in the ultrafast demagnetization process in magnetically inhomogeneous samples. Making use of at least partially circularly polarized radiation, the imaging of magnetic domains was also possible at the $L$ and $M$-edges of cobalt.

Future experiments will use double FEL pulse schemes, using the FEL for pumping as well as for probing. The FEL pulse can be split by an optical delay-line device for longer delay times up to the nanosecond range. A different method to generate double FEL pulses, even with slightly different energies, is to seed different parts of the electron bunch and therefore obtain two independent pulses with a delay time of a few hundreds of femtoseconds, as shown at FERMI [32]. Furthermore, $\mathrm{THz}$ radiation can serve as a pump. Here, FLASH offers the possibility to use a $\mathrm{THz}$ pulse created by the same electron bunch as the FEL radiation [33]. Hence, the jitter is minimal and measurements can be performed under phase-stable conditions.

Many FEL projects are under construction worldwide. One of those, the FLASH II project [34], currently under construction in Hamburg, will offer higher energies up to the $L$-edges of the transition metals of interest $(\mathrm{Fe}, \mathrm{Co}, \mathrm{Ni})$ using the third harmonic of the undulator and an option for providing circularly polarized radiation is foreseen. The direct availability of circularly polarized radiation will not only further promote magnetic FTH imaging experiments but also will provide circularly polarized radiation independently of dichroic transitions.

\section{ACKNOWLEDGEMENT}

The authors would like to thank the scientific and technical support teams at FLASH, 
LCLS, and FERMI for their help during the experiments. The experiments would not have been possible without the experience gained with experiments at ESRF ID08, BESSY- II, and Petra III P04. We would like to thank the beamline staff for their support.

Financial support by the DFG within SFB 668 and from the BMBF under Contract No. 05K10KTB/FSP-301 and 05K10GU4/FSP-301 is gratefully acknowledged. This work has been supported by the excellence cluster "The Hamburg Centre for Ultrafast Imaging - Structure, Dynamics, and Control of Matter at the Atomic Scale" of the DFG. The French participants acknowledge support from the CNRS through the PEPS SASELEX and J.L., J.G., P.Z., and B.V. acknowledge support from the French ANR via the FEMTO-X- MAG project. B.T. And K.L. Acknowledge support from the ERASMUS

Mundus exchange program. S. E. and G. G. acknowledge support in the Helmholtz Virtual Institute "Dynamic Pathways in Multidimensional Landscapes" (VH-VI-419).

\section{LITERATURE}

[1] C. Gutt, L. M. Stadler, S. Streit-Nierobisch, A. P. Mancuso, A. Schropp, B. Pfau, C. M. Günther, R. Könnecke, J. Gulden, B. Reime, et al., Phys. Rev. B 79, 212406 (2009).

[2] C. Gutt, S. Streit-Nierobisch, L.-M. Stadler, B. Pfau, C. M. Günther, R. Könnecke, R. Frömter, A. Kobs, D. Stickler, H. P. Oepen, et al., Phys. Rev. B 81, 100401(R) (2010).

[3] B. Pfau, S. Schaffert, L. Müller, C. Gutt, A. Al-Shemmary, F. Büttner, R. Delauny, S. Düsterer, S. Flewett, R. Frömter, et al., Nat. Commun. 3, 1100 (2012).

[4] C. E. Graves, A. H. Reid, T. Wang, B. Wu, S. de Jong, K. Vahaplar, I. Radu, D. P. Bernstein, M. Messerschmidt, L. Müller, et al., Nat. Mater. 12, 293 (2013).

[5] T. Wang, D. Zhu, B. Wu, C. Graves, S. Schaffert, T. Rander, L. Müller, B. Vodungbo, C. Baumier, D. P. Bernstein, et al., Phys. Rev. Lett. 108, 267403 (2012).

[6] L. Müller, C. Gutt, B. Pfau, S. Schaffert, J. Geilhufe, F. Büttner, J. Mohanty, S. Flewett, R. Treusch, S. Düsterer, et al., Phys. Rev. Lett. 110, 234801 (2013).

[7] G. Schütz, W. Wagner, W. Wilhelm, and P. Kienle, Phys. Rev. Lett. 58, 737 (1987).

[8] B. T. Thole, P. Carra, F. Sette, and G. van der Laan, Phys. Rev. Lett. 68, 1943 (1992).

[9] O. Hellwig, G. P. Denbeaux, J. B. Kortright, and E. E. Fullerton, Physica B 336, 136 (2003). 
[10] S. Eisebitt, J. Lüning, W. F. Schlotter, M. Lörgen, O. Hellwig, W. Eberhard, and J. Stöhr, Nature 432, 885 (2004).

[11] T. Hauet, C. M. Günther, B. Pfau, M. E. Schabes, J.-U. Thiele, R. L. Rick, P. Fischer, S. Eisebitt, and O. Hellwig, Phys. Rev. B 77, 184421 (2008).

[12] S. Streit-Nierobisch, D. Stickler, C. Gutt, L. M. Stadler, H. Stillrich, C. Menk, R. Frömter, C. Tieg, O. Leupold, H. P. Oepen, et al., J. Appl. Phys. 106, 083909 (2009).

[13] E. Beaurepaire, J.-C. Merle, A. Daunois, and J.-Y. Bigot, Phys. Rev. Lett. 76, 4250 (1996).

[14] A. Kirilyuk, A. V. Kimel, and T. Rasing, Rev. Mod. Phys. 82, 2732 (2010).

[15] R. W. Schoenlein, S. Chattopadhyay, H. H. W. Chong, T. E. Glover, P. A. Heimann, C. V. Shank, A. A. Zholents, and M. S. Zolotorev, Science 287, 2237 (2000).

[16] C. Stamm, T. Kachel, N. Pontius, R. Mitzner, T. Quast, K. Holldack, S. Khan, C. Lupulescu, E. F. Aziz, M. Wietstruk, et al., Nat. Mater. 6, 740 (2007).

[17] C. Boeglin, E. Beaurepaire, V. Halté, V. López-Flores, C. Stamm, N. Pontius, H. A. Dürr, and J.-Y. Bigot, Nature 465, 458 (2010).

[18] C. M. Günther, B. Pfau, R. Mitzner, B. Siemer, S. Roling, H. Zacharias, O. Kutz, I. Rudolph, D. Schondelmaier, R. Treusch, et al., Nat. Phot. 5, 99 (2011).

[19] L. Müller, C. Gutt, S. Streit-Nierobisch, M. Walther, S. Schaffert, B. Pfau, J. Geilhufe, F. Buettner, S. Flewett, C. M. Günther, et al., Rev. Sci. Instrum. 84, 0139006 (2013).

[20] K. Vogt, S. Schultheiss, S. J. Hemsdoerfer, P. Pirro, A. A. Serga, and B. Hillebrands, Appl. Phys. Lett. 95, 182508 (2009).

[21] P. J. Metaxas, J. P. Jamet, A. Mougin, M. Cormier, J. Ferré, V. Baltz, B. Rodmacq, B. Dieny, and R. L. Stamps, Phys. Rev. Lett. 99, 217208 (2007).

[22] I. M. Miron, T. Moore, H. Szambolics, L. D. Buda-Prejbeanu, S. Auffret, B. Rodmacq, S. Pizzini, J. Vogel, M. Bonfim, A. Schuhl, et al., Nat. Mater 10, 419 (2011).

[23] M. Battiato, K. Carva, and P. M. Oppeneer, Phys. Rev. Lett. 105, 027203 (2010).

[24] B. Pfau, C. M. Günther, R. Könnecke, E. Guehrs, O. Hellwig, W. F. Schlotter, and S. Eisebitt, Opt. Express 18, 13608 (2010).

[25] S. Eisebitt, M. Lörgen, W. Eberhard, J. Lüning, J. Stöhr, C. T. Rettner, O. Hellwig, E. E. Fullerton, and G. P. Denbeaux, Phys. Rev. B 68, 104419 (2003).

[26] W. F. Schlotter, J. J. Turner, M. Rowen, P. Heimann, M. Holmes, O. Krupin, M. Messerschmidt, S. Moeller, J. Krzywinski, R. Soufli, et al., Rev. Sci. Instrum. 83, 043107 (2012).

[27] F. Capotondi, E. Pedersoli, N. Mahne, R. H. Menk, G. Passos, L. Raimondi, C. Svetina, G. 
Sandrin, M. Zangrando, M. Kiskinova, et al., Rev. Sci. Instr. 84 (2013).

[28] E. Pedersoli, F. Capotondi, D. Cocco, M. Zangrando, B. Kaulich, R. H. Menk, A. Locatelli, T. O. Mentes, C. Spezzani, G. Sandrin, et al., Rev. Sci. Instrum. 82, 043711 (2011).

[29] S. Schaffert, B. Pfau, J. Geilhufe, C. M. Günther, C. M. Schneider, C. von Korff- Schmising, and S. Eisebitt, New J. Phys. In print, (2013).

[30] H. N. Chapman, S. P. Hau-Riege, M. J. Bogan, S. Bajt, A. Barty, S. Boutet, S. Marchesini, M. Frank, B. W. Woods, W. H. Benner, et al., Nature 448, 676 (2007).

[31] S.-K. Son, L. Young, and R. Santra, Phys. Rev. A 83, 033402 (2011).

[32] E. Allaria, F. Bencivenga, R. Borghes, F. Capotondi, D. Castronovo, P. Charalambous, P. Cinquegrana, B. Danailov, G. D. Ninno, A. Demidovich, et al., Nature Comm. 4, 2476 (2013). (to appear, check reference)

[33] M. Gensch, L. Bittner, A. Chesnov, H. Delsim-Hashemi, M. Drescher, B. Faatz, J. Feldhaus, U. Fruehling, G. A. Geloni, C. Gerth, et al., Infrared Phys. Technol. 51, 423 (2008),

[34] B. Faatz, N. Baboi, V. Ayvazyan, V. Balandin, W. Decking, S. Duesterer, H. J. Eckoldt, J. Feldhaus, N. Golubeva, K. Honkavaara, et al., Nucl. Instr. Meth. Phys. Res. A 635, S2 (2011). 


\section{FIGURES}



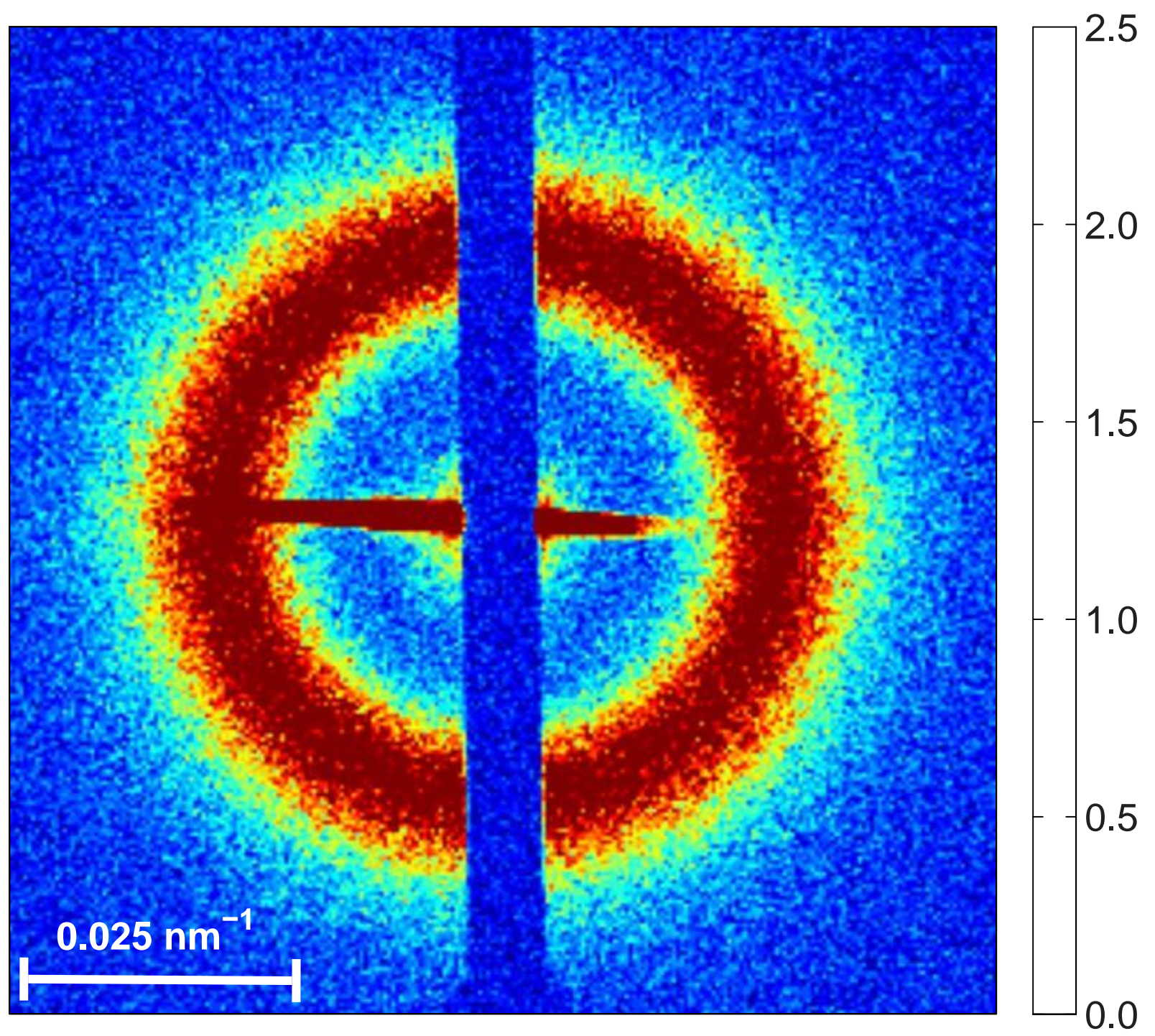

Figure 1. Resonant magnetic small-angle scattering pattern of a cobalt/platinum multilayer with perpendicular magnetic anisotropy, prepared in the disordered maze-domain state. The pattern is recorded using a single FEL shot of $30 \mathrm{fs}$ duration at the cobalt $M$-edge at $59.6 \mathrm{eV}$. The pulse intensity of $2 \mu \mathrm{J}$ corresponds to a fluence of $4 \mathrm{~mJ} / \mathrm{cm}^{2}$ and a power density of $1.3 \times 10^{11} \mathrm{~W} / \mathrm{cm}^{2}$. The color scale indicates the scattering intensity. Reprinted from [2] copyright (2010) by The American Physical Society. 

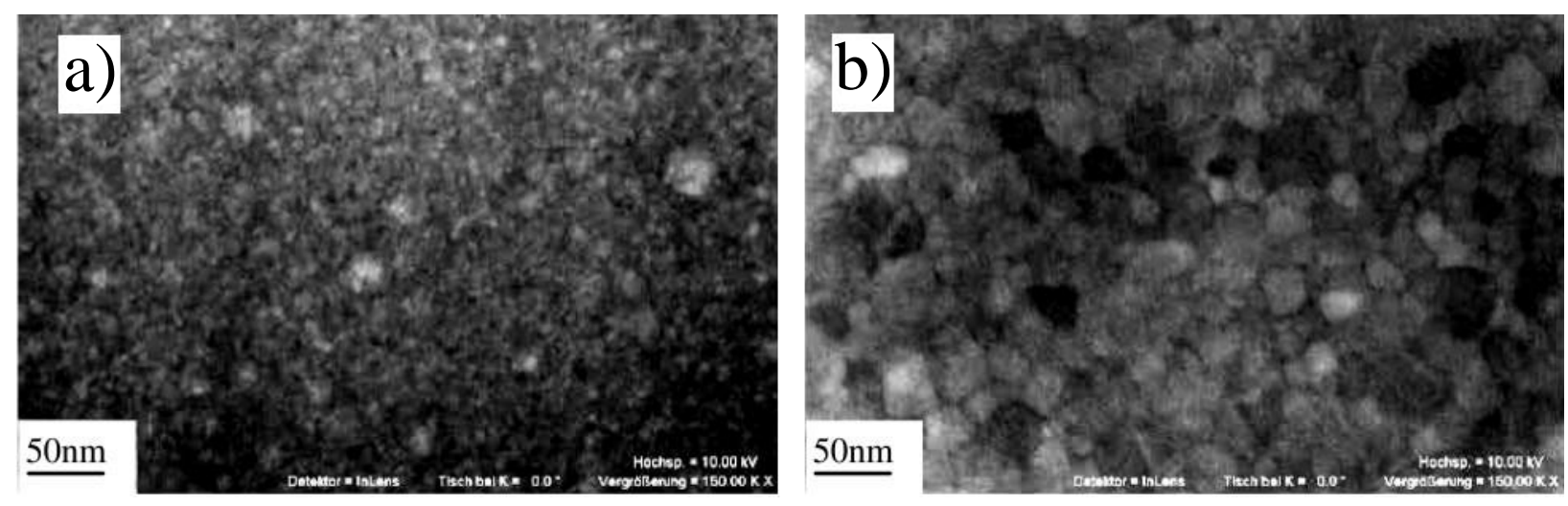

Figure 2. Scanning-electron micrograph of a non-irradiated (a) and an FEL irradiated (b) Co/Pt multilayer sample. The FEL irradiation leads to growth of the crystal grains. 


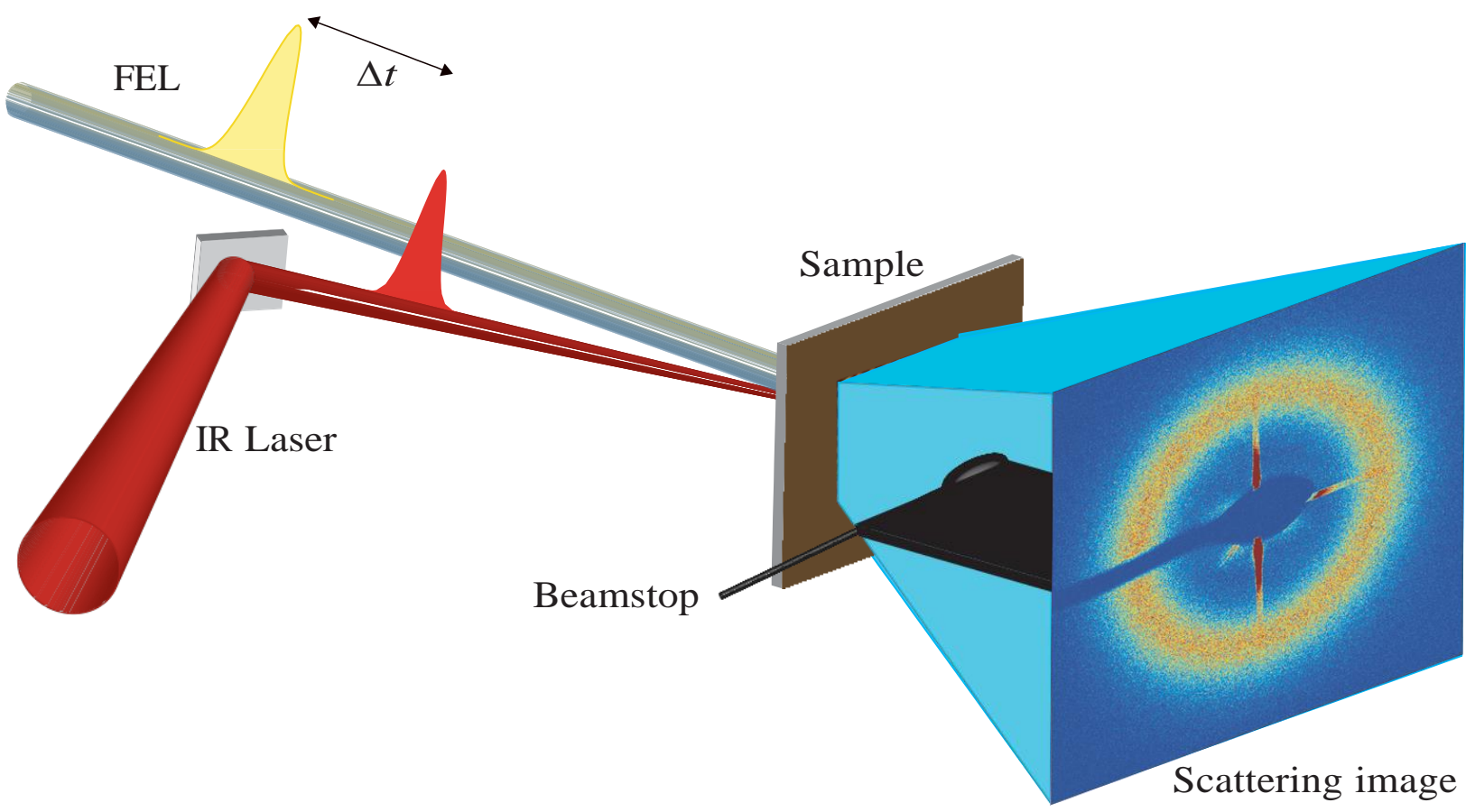

Figure 3. General design of an optical-pump-FEL-probe experiment. Both laser pulses are incident on the sample with an adjustable time delay $\Delta t$. The small angle scattering signal of the magnetic sample is detected by an area detector. Reprinted with permission from [19], copyright (2013) AIP Publishing LLC. 

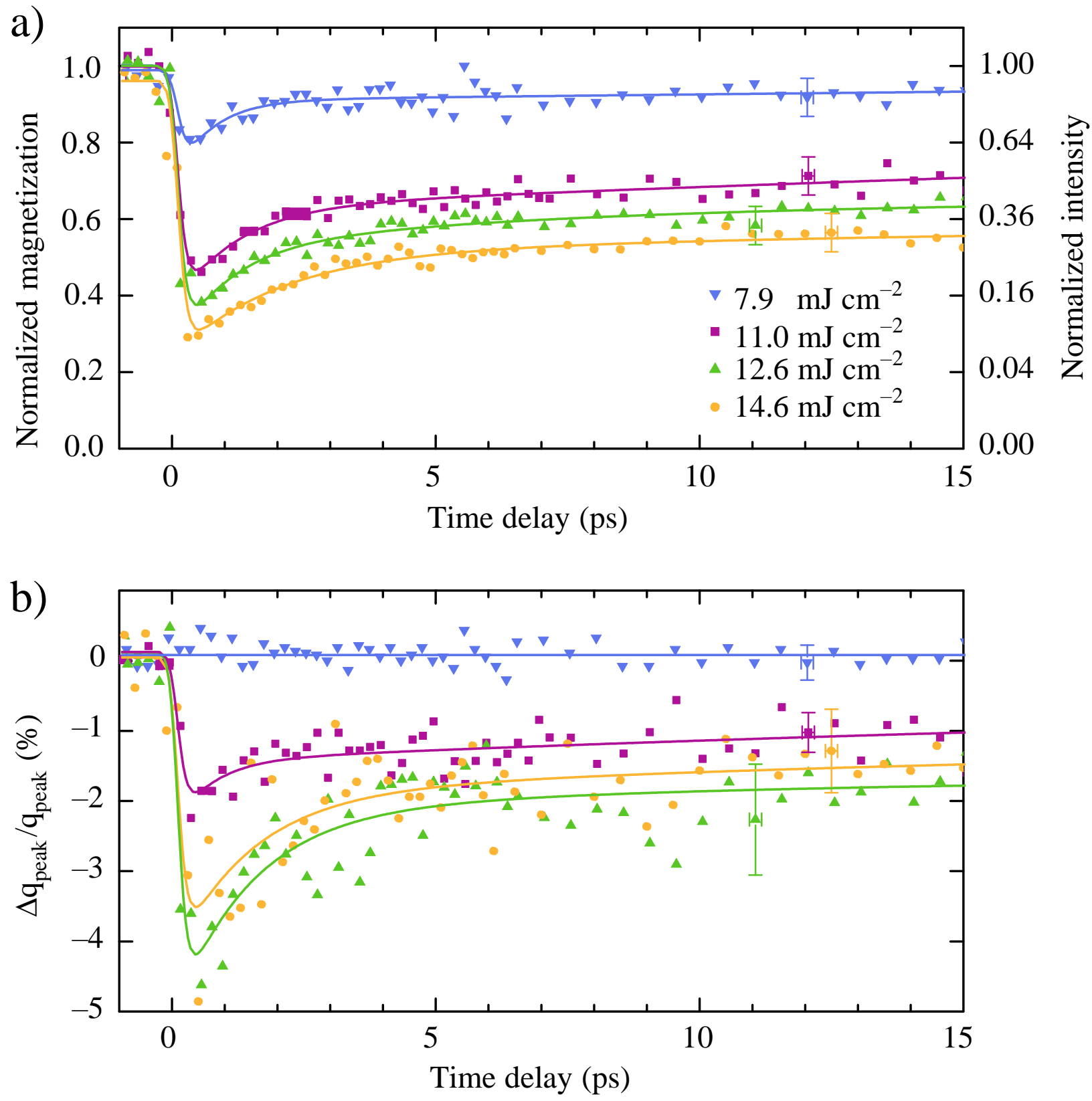

Figure 4. Ultrafast demagnetization (a) and peak shift of the maximum intensity position of the radially integrated small-angle scattering ring (b). The solid lines are fits using the sum of three exponentials convolved with the experimental time resolution. The time constants of the exponentials are the demagnetization time and the time constants of the fast and slow recovery. Reproduced from [3]. 


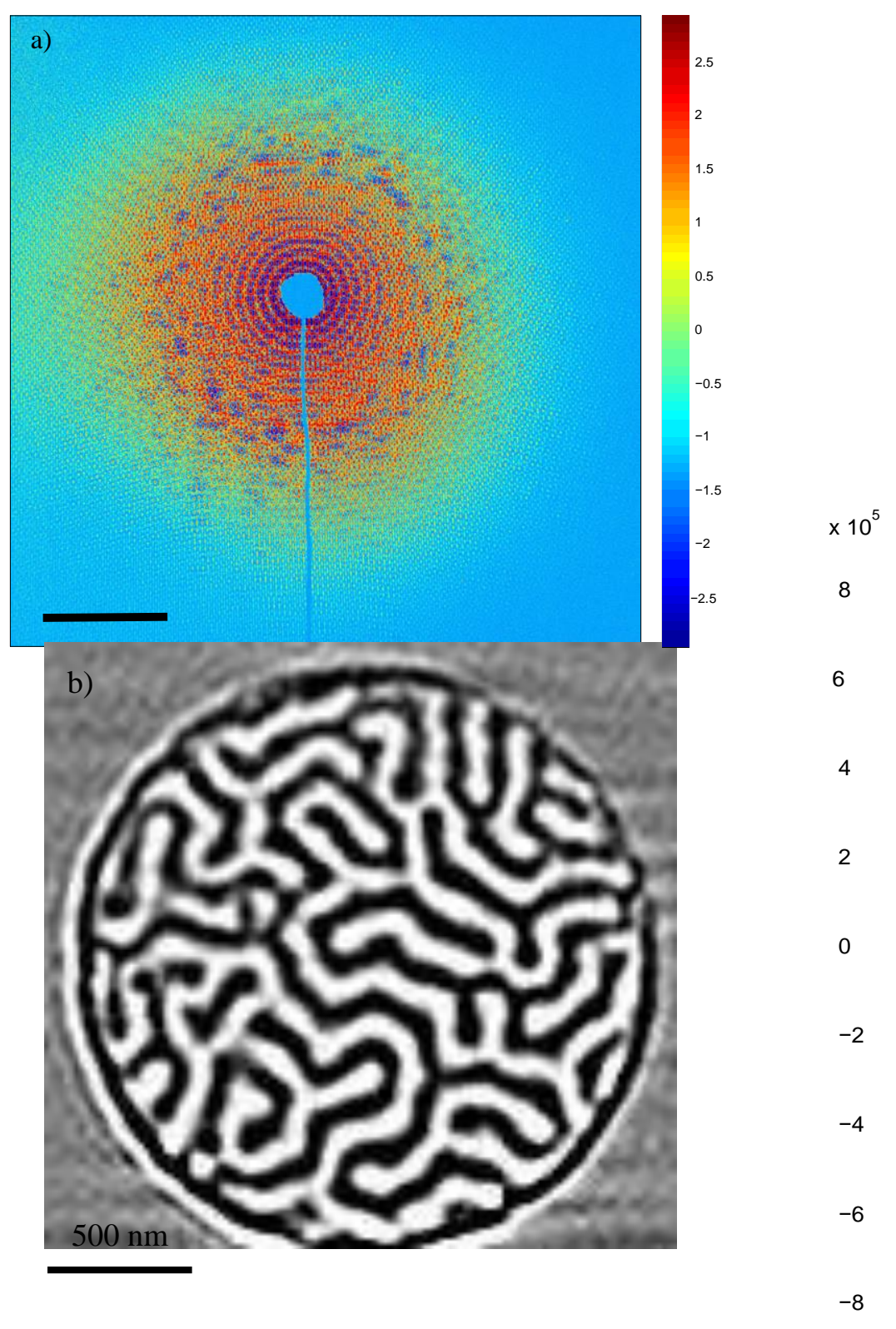

Figure 5. (a) Magnetic hologram calculated as the difference of holograms taken with left and right circularly polarized radiation at FERMI. The photon energy was tuned to the cobalt $M$-edge at $59.6 \mathrm{eV}(20.8 \mathrm{~nm})$ and the pulse length is around $80 \mathrm{fs}$. The color scale shows the scattering intensity on logarithmic scale preserving the sign resulting from the substraction. (b) Real-space reconstruction of the hologram. The domains with a typical width of $70 \mathrm{~nm}$ can be resolved clearly. 

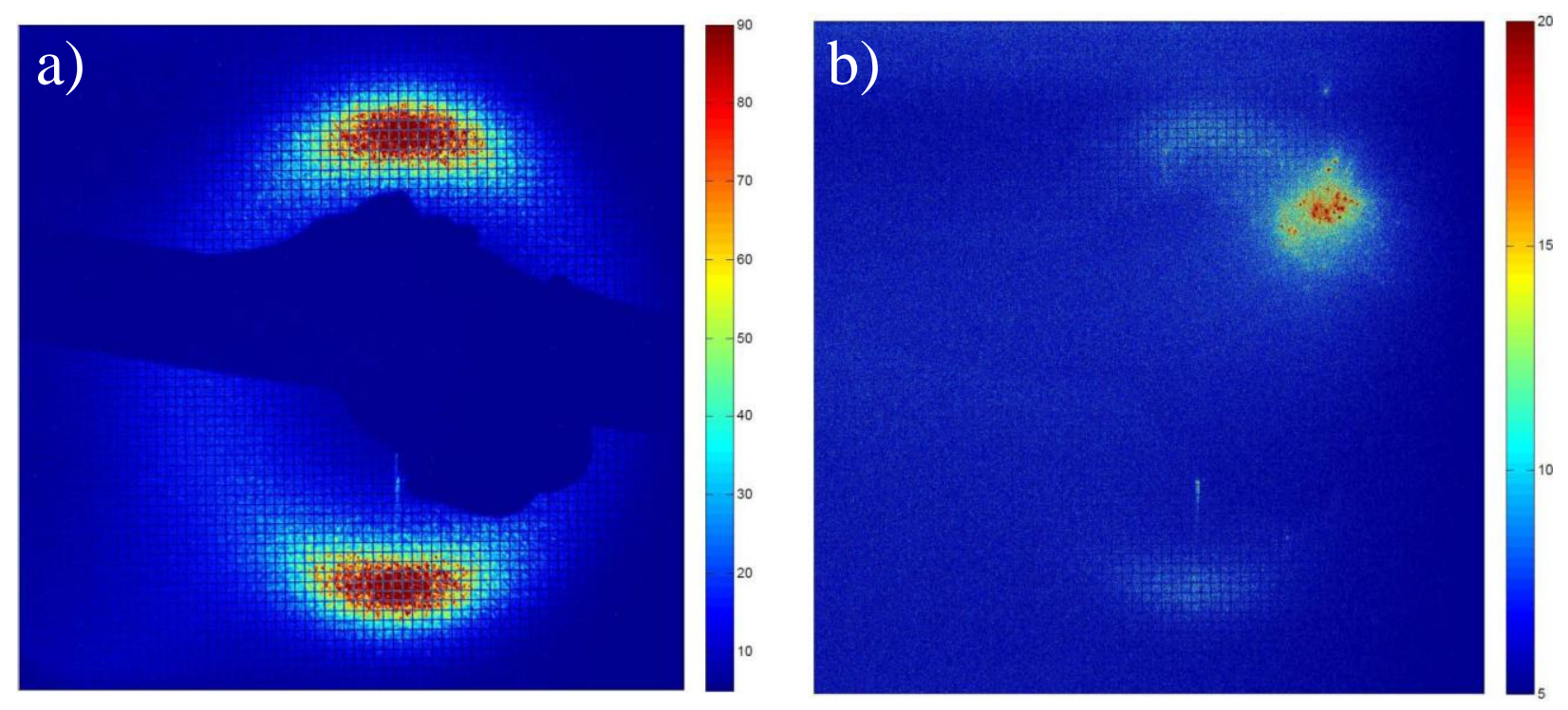

Figure 6. Scattering images of a magnetic sample with domains partially aligned into stripes. The FEL is tuned to the Co $M$ edge at $20.8 \mathrm{~nm}$. (a) Scattering pattern from 1000 low-fluence shots with a total flux of $7.5 \mathrm{~J} / \mathrm{cm}^{2}$. (b) Single-shot scattering pattern from a single pulse with a fluence of $5 \mathrm{~J} / \mathrm{cm}^{2}$. The scale bar indicates the number of photons per pixel. The scattering intensity is decreased by a factor of 20 in the high fluence case. Furthermore, a parasitic signal originating from the destruction of the sample is visible. Reprinted from [6] copyright (2013) by The American Physical Society. 